\title{
Novel Experience of Contrast-Enhanced Ultrasonography to Differentiate Between Renal Cysts and Renal Cell Carcinoma
}

\author{
Jun-Koo Kang ${ }^{1}$, Hyejin Cheon ${ }^{2}$, Yun-Sok Ha ${ }^{1}$, Jae-Wook Chung ${ }^{1}$ \\ ${ }^{1}$ Department of Urology, School of Medicine, Kyungpook National University, Daegu, Korea \\ ${ }^{2}$ Department of Radiology, School of Medicine, Kyungpook National University, Daegu, Korea
}

\begin{abstract}
We report our first experience with the use of contrast-enhanced ultrasonography (CEUS) to differentiate between a complicated hemorrhagic renal cyst and a cystic renal cell carcinoma in a 50-year-old man diagnosed with autosomal dominant polycystic kidney disease undergoing hemodialysis for end-stage renal disease. CEUS could successfully differentiate between a complicated hemorrhagic renal cyst and a cystic renal cell carcinoma, as opposed to computed tomography (CT) or magnetic resonance imaging (MRI), which could not distinguish between the 2 disease conditions. CEUS is comparable diagnostic tool as CT or MRI to distinguish between benign and malignant cystic renal masses. (Korean J Urol Oncol 2018;16:42-45)
\end{abstract}

Key Words: Contrast $\cdot$ Cyst $\cdot$ Enhance $\cdot$ Renal cell carcinoma $\cdot$ Ultrasonography

Complicated renal cysts containing a hematoma or proteinaceous material are often indistinguishable from cystic renal cell carcinoma (RCC) using computed tomography (CT) or magnetic resonance imaging (MRI) examination. While complicated renal cysts belonging to the Bosniak classification III or IV require surgical treatment, Bosniak classification IIF cysts require regular long-term follow-up. However, in those at high risk of surgery, accurate diagnostic methods are needed to appropriately screen patients to determine their suitability as surgical candidates. We describe our first-time/unique experience with use of contrast-enhanced ultrasonography (CEUS) as a diagnostic modality for a cystic renal mass.

Received March 29, 2018, Revised April 3, 2018,

Accepted April 3, 2018

Corresponding Author: Jae-Wook Chung

Department of Urology, School of Medicine, Kyungpook National University, 680 Gukchaebosang-ro, Jung-gu, Daegu 41944, Korea

E-mail: jeus119@hanmail.net

Tel: +82-53-200-2166, Fax: +82-53-321-3207

ORCID code: https://orcid.org/0000-0002-1055-2357

\section{CASE REPORT}

A 50-year-old man presented to the outpatient clinic of our urology center. He reported a history of autosomal dominant polycystic kidney and had been receiving hemodialysis at a local clinic since 1989 for the management of end-stage renal disease. He had been admitted to the local clinic because of a fever that had developed a month prior to presentation. His fever was identified as fever of unknown origin, and an abdominal CT revealed a complicated renal cyst. He was referred to our clinic for further investigations and to rule out malignancy. Upon admission, his vital signs were stable, he was afebrile and laboratory tests were within reference range except elevated levels of blood urea nitrogen $(40.8 \mathrm{mg} / \mathrm{dL})$ and creatinine $(6.25 \mathrm{mg} / \mathrm{dL})$. We performed a dynamic CT of the kidneys to check for malignancy and adjusted his hemodialysis schedule immediately after the CT to reduce the adverse effect associated with the use of the contrast agent. The dynamic CT of the kidneys revealed a heterogeneous slightly hyperdense mass measuring $7.7 \mathrm{~cm}$ with linear mural calcifications noted

cc) (7) (5) This is an Open Access article distributed under the terms of the Creative Commons Attribution Non-Commercial License (http://creativecommons.org/licenses/by-nc/4.0/) which permits unrestricted non-commercial use, distribution, and reproduction in any medium, provided the original work is properly cited. 2018 (C) Copyright The Korean Urological Oncology Society and The Korean Prostate Society. All Rights Reserved. 
at the lower pole of the left kidney that did not show significant enhancement on contrast-enhanced CT images (Fig. 1). It is often difficult to differentiate between a papillary RCC and a benign hyperdense cyst containing an organizing hematoma or proteinaceous material because in several cases, papillary RCCs do not show enhancement on CT. A radiology consultation was performed, and he was recommended to undergo contrast-enhanced MRI assessment of the kidneys.
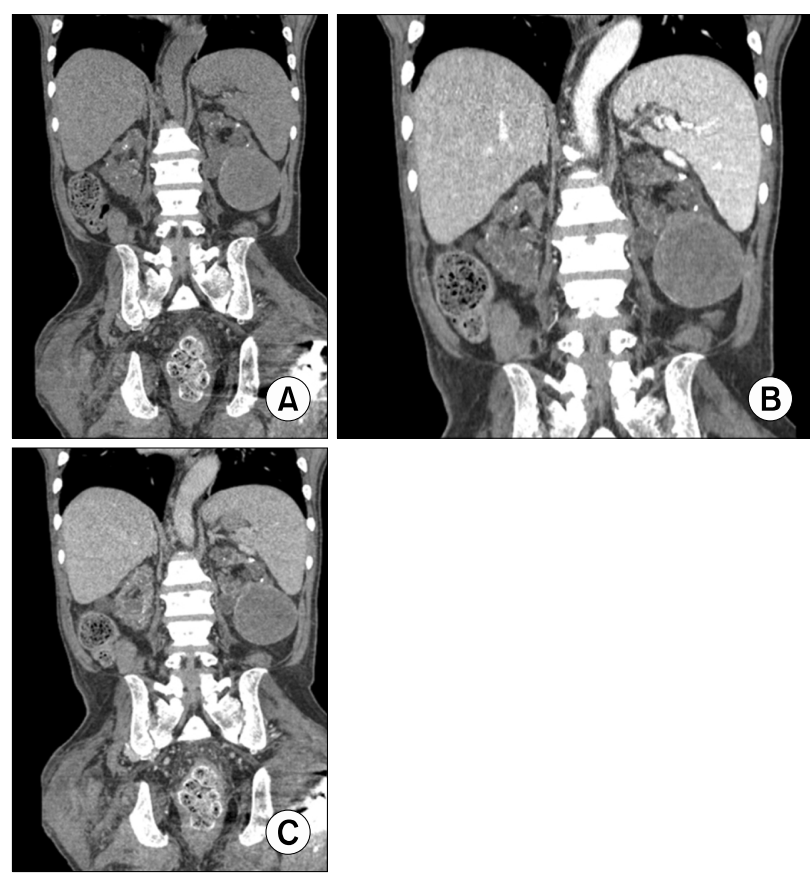

B.

Fig. 1. Coronal reformatted non contrast computed tomography (CT) image (A) revealed a hyperdense mass at the lower pole of the left kidney, measuring $7.7 \mathrm{~cm}$ with linear mural calcifications and contrast enhanced CT images in corticomedullary phase (B) and nephrographic phase (C) showed no definite enhancement.
A week later, we performed MRI of the kidneys using a 3-T MRI scanner (GE DISCOVERY 750, Milwaukee, WI, USA). The study showed a $\mathrm{T} 1$ hyperintense mass at the lower pole of the left kidney, which measured $7.7 \mathrm{~cm}$ with a dark peripheral portion and a central cystic portion on T2-weighted images. The mass revealed no enhancement on enhanced T1-weighted images on visual assessment (Fig. 2). However, subtracted MR images showed thick peripheral enhancement at the upper margin of the lesion, which was determined to be a misregistration artifact secondary to patient motion because of variability in/inappropriate breath-holding by patients. He was recommended to undergo CEUS to evaluate lesion enhancement to help distinguish between a papillary RCC and a hemorrhagic cyst.

Approximately 2 weeks later, the patient underwent CEUS (Philips EPIQ 7 with sulfur hexafluoride blood-pool agent [SonoVue, Bracco S.p.A., Milano, Italy]) (Fig. 3). CEUS revealed no enhancement in any portion of the left kidney. The patient was reassured regarding the very low chance of malignancy and we opted to continue management with CEUS performed every 3 or 6 months.

Follow-up CEUS was performed 6 months later. The result was same: there was no enhancement in any portion of the left kidney. We are planning to perform 1-year follow-up CEUS.

\section{DISCUSSION}

The incidence rate of renal cysts noted in clinical practice is approximately $50 \%$ in patients aged $>50$ years. ${ }^{1}$ Most lesions are benign simple cysts; however, complex and multifocal cystic renal lesions are also relatively common. ${ }^{2}$ Differentiating between benign cysts and cystic RCCs requiring specific onco-
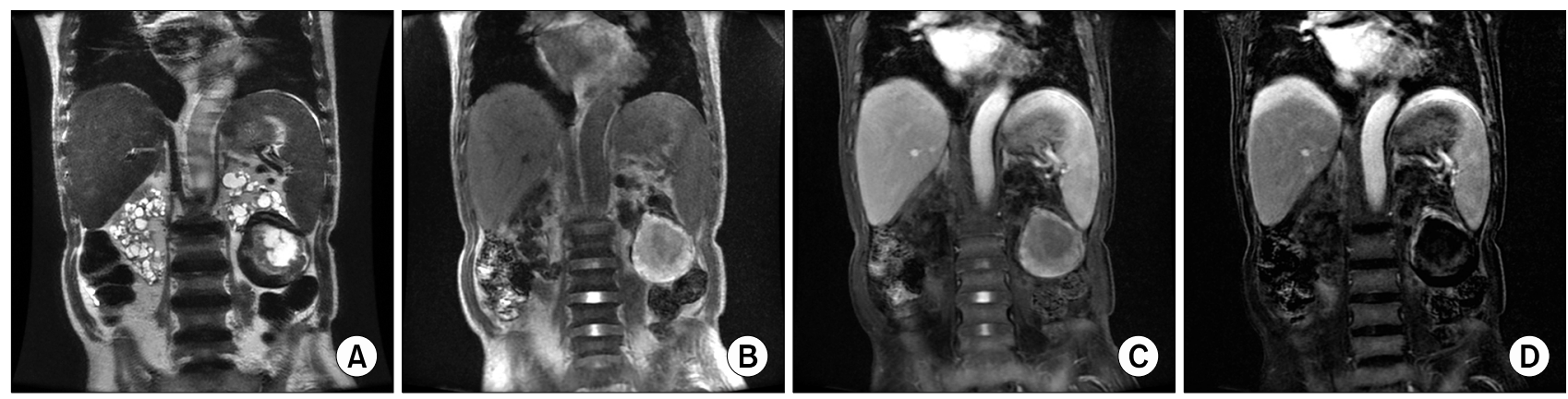

Fig. 2. Magnetic resonance imaging (MRI) showing a complex cystic mass at the lower pole of the left kidney measuring $7.7 \mathrm{~cm}$ with a high signal intensity on T1-weighted image (A) and a dark peripheral portion and central cystic portion (B). (C) Contrast-enhanced T1-weighted image showing no definite enhancement on visual assessment compared to a T1-weighted image. (D) Subtracted T1-weighted image showing a misregistration artifact at the subdiaphgram and periphery of the cystic mass. 

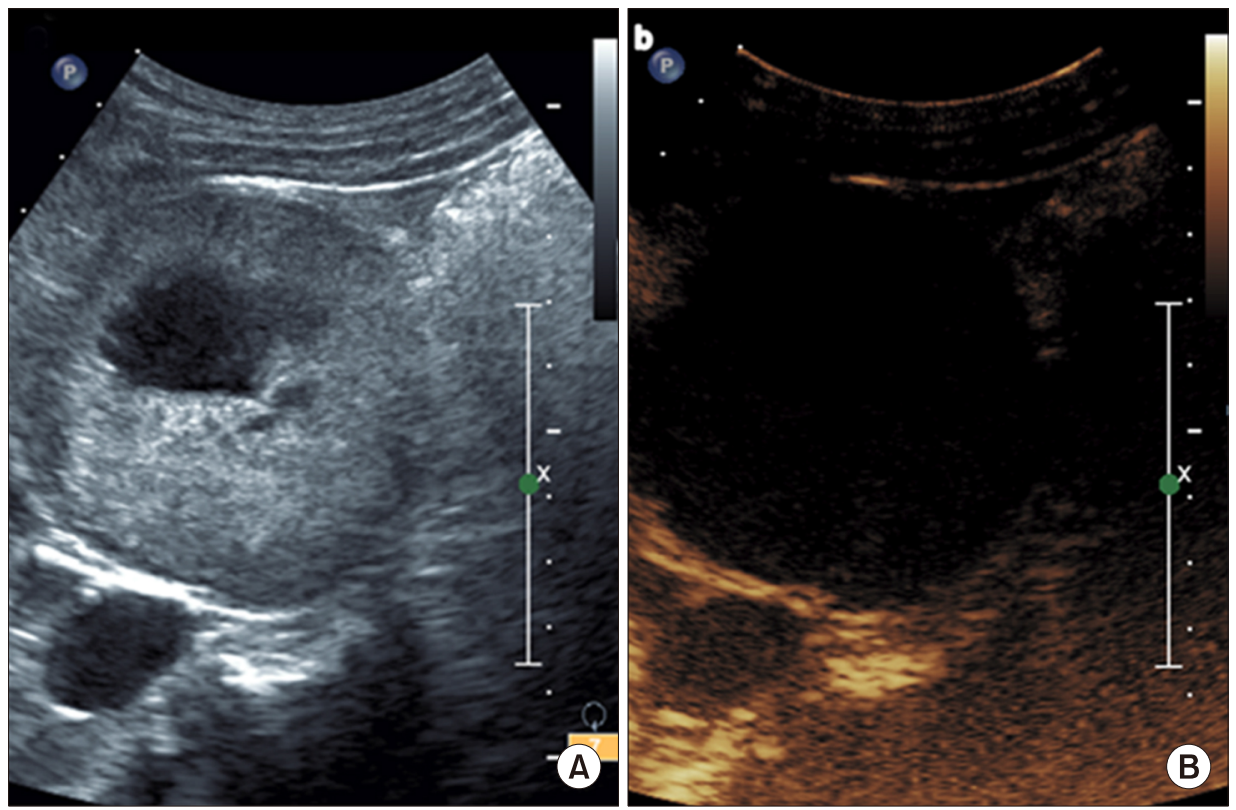

Fig. 3. Single image from cine clips: B-mode ultrasound (A) revealed hyperechoic lesion with central cystic portion and contrast-enhanced ultrasonography with low mechanic index technique (B) revealed no enhancement observed in any section of the left kidney.

logical care is a diagnostic challenge. ${ }^{3}$ These cystic cancers constitute approximately $10 \%$ of renal cancers. Notably, in ESRD patients, the incidence of renal malignancies is markedly increased related to the development of acquired cystic kidney disease. ${ }^{4}$

CEUS is the newest diagnostic tool available to detect vascularization using intravenous contrast agents containing microbubbles. ${ }^{5,6}$ CEUS software allows the operator to subtract the background soft tissue and visualize a contrast-enhancement-only image. A narrow width of the ultrasound (US) beam enables improved visualization of vascularity in small structures such as septations or small mural nodules. Additionally, Chang et $\mathrm{al}^{7}{ }^{7}$ have demonstrated that the sensitivity of CEUS compared to tissue diagnosis and/or follow-up imaging is high and comparable to the reported sensitivities of CT and MRI among patients with and without chronic kidney disease. High sensitivity provides a high certainty that a negative test in an individual means that the individual does not have the disease, which essentially rules out malignancy. Several recent reports have demonstrated that contrast-enhanced US showed a sensitivity of $100 \%$ for evaluation of indeterminate renal masses. ${ }^{8}$

In addition, ultrasound contrast agents are not nephrotoxic and can be safely used in patients with renal impairment. Therefore, CEUS can provide a safe first-line tool for investigating renal anomalies in the patients with acute kidney injury or chronic kidney disease. ${ }^{9}$

In our patient described in this report, we suspected that the hyperdense mass could have been a benign hyperdense chronic hemorrhagic cyst or a malignant cyst such as a cystic RCC with hemorrhage or a papillary RCC without enhancement because papillary RCCs are known to not show enhancement on radiological examination, indicating that enhancement alone is insufficient/inaccurate to differentiate between papillary RCCs and hyderdense cysts on CT examination. ${ }^{10}$ Although MRI is more sensitive than $\mathrm{CT}$ examination in detecting enhancement and can aid qualitative assessment using subtraction images, MRI examination is associated with limitations such as misregistration artifacts secondary to variability in breath-holding by patients and subsequent motion, as well as the risk of nephrogenic systemic fibrosis in chronic kidney disease patients.

CEUS is the most suitable and ideal imaging modality to evaluate internal enhancement observed in a hyperdense mass that shows no enhancement. Thus, we concluded that the hyperdense mass observed in our patient was a benign hemorrhagic cyst.

A limitation of this study was that we did not perform histopathological examination that is usually performed following surgical removal of cysts. General medical condition of the patient is extremely poor so we cannot perform operation. Therefore, it is difficult to conclude that the kidneys were completely free of malignancy. And follow-up period was too short (6 months). Furthermore, CT and MRI already have shown the low possibility of malignancy so we cannot generalize that CEUS is better than CT or MRI with only this one case. 
Prospective, randomized, multicenter trials including large number of lesions are needed in the future.

Regardless of its limitations, CEUS should be considered a useful diagnostic tool to aid in counseling patients with not only complex cystic renal masses but also those who are categorized as high-risk surgical candidates.

\section{CONFLICT OF INTEREST}

The authors claim no conflicts of interest.

\section{REFERENCES}

1. McGuire BB, Fitzpatrick JM. The diagnosis and management of complex renal cysts. Curr Opin Urol 2010;20:349-54.

2. Wood CG 3rd, Stromberg LJ 3rd, Harmath CB, Horowitz JM, Feng C, Hammond NA, et al. CT and MR imaging for evaluation of cystic renal lesions and diseases. Radiographics 2015;35:125-41.

3. Defortescu G, Cornu JN, Béjar S, Giwerc A, Gobet F, Werquin $\mathrm{C}$, et al. Diagnostic performance of contrast-enhanced ultrasonography and magnetic resonance imaging for the assess- ment of complex renal cysts: a prospective study. Int J Urol 2017;24:184-9.

4. Rahbari-Oskoui F, O'Neill WC. Diagnosis and management of acquired cystic kidney disease and renal tumors in ESRD patients. Semin Dial 2017;30:373-9.

5. Greis C. Summary of technical principles of contrast sonography and future perspectives. Radiologe 2011;51:456-61.

6. Greis C. Ultrasound contrast agents as markers of vascularity and microcirculation. Clin Hemorheol Microcirc 2009;43:1-9.

7. Chang EH, Chong WK, Kasoji SK, Fielding JR, Altun E, Mullin LB, et al. Diagnostic accuracy of contrast-enhanced ultrasound for characterization of kidney lesions in patients with and without chronic kidney disease. BMC Nephrol 2017;18:266.

8. Barr RG, Peterson C, Hindi A. Evaluation of indeterminate renal masses with contrast-enhanced US: a diagnostic performance study. Radiology 2014;271:133-42.

9. Jakobsen JA, Oyen R, Thomsen HS, Morcos SK; Members of Contrast Media Safety Committee of European Society of Urogenital Radiology (ESUR). Safety of ultrasound contrast agents. Eur Radiol 2005;15:941-5.

10. Egbert ND, Caoili EM, Cohan RH, Davenport MS, Francis IR, Kunju LP, et al. Differentiation of papillary renal cell carcinoma subtypes on CT and MRI. AJR Am J Roentgenol 2013;201:347-55. 